\title{
RIGHT ADJOINT FOR THE SMASH PRODUCT FUNCTOR
}

\author{
FRANCESCA CAGLIARI
}

(Communicated by Andreas R. Blass)

\begin{abstract}
The smash-product functor $(-) \wedge\left(X, x_{0}\right)$ in the category Top $\underline{\text { Top }}_{*}$ of pointed topological spaces has a right adjoint for any choice of the base point $x_{0}$, if and only if the topological space $X$ is quasi-locally compact, that is, if and only if the product functor $(-) \times X$ has a right adjoint in the category Top of topological spaces.
\end{abstract}

\section{INTRODUCTION}

A space $X$ is cartesian in the category of topological spaces and continuous maps if the product functor $(-) \times X$ has a right adjoint. This means that there exists a proper and admissible topology on the space of maps $Y^{X}$ between $X$ and $Y$ (for any topological space $Y$ ) [D]. Cartesian objects in Top were characterized by Day and Kelly $[\mathrm{D}-\mathrm{K}]$. They are the quasi-locally compact spaces $[\mathrm{H}-\mathrm{L}]$.

The problem of the existence of a proper and admissible topology on the function space $\left(Y, y_{0}\right)^{\left(X, x_{0}\right)}$ consisting of the maps of $Y^{X}$ preserving base points is related to the adjointness of the smash-product functor. It is known that this functor has a right adjoint whenever $X$ is locally compact and Hausdorff; in this case the topology on $\left(Y, y_{0}\right)^{\left(X, x_{0}\right)}$ is the compact open topology [M].

In this paper, it is proved that the spaces $\left(X, x_{0}\right)$ for which the functor $(-) \wedge$ $\left(X, x_{0}\right)$ has a right adjoint are exactly the spaces $X$ which are cartesian in Top, independently of the choice of $x_{0}$. That is, the existence of a proper and admissible topology on $\left(Y, y_{0}\right)^{\left(X, x_{0}\right)}$ for any $\left(Y, y_{0}\right)$ is equivalent to the existence of a proper and admissible topology on the whole space of maps from $X$ to $Y$, for any $Y$.

\section{SMASH-PRODUCT AND ADJUNCTION}

We can consider, in $\underline{\mathbf{T o p}}_{*}$, the endofunctor $(-) \wedge\left(X, x_{0}\right)$ and ask when it has a right adjoint. When it exists we will call it $\mathcal{G}_{\left(X, x_{0}\right)}$.

In the case of $X$ cartesian in Top, we indicate by $Y^{X}$ the power object and by $\left(Y, y_{0}\right)^{\left(X, x_{0}\right)}$ the subspace of $Y^{X}$ given by $\left\{f \in Y^{X} \mid f\left(x_{0}\right)=y_{0}\right\}$ with base point the constant $y_{0}$-valued map.

Theorem 1. If $X$ is cartesian in Top, then $(-) \wedge\left(X, x_{0}\right)$ has a right adjoint, for every $x_{0}$ in $X$. Moreover $\mathcal{G}_{\left(X, x_{0}\right)}\left(Y, y_{0}\right)=\left(Y, y_{0}\right)^{\left(X, x_{0}\right)}$.

Received by the editors November 3, 1993 and, in revised form, August 19, 1994

1991 Mathematics Subject Classification. Primary 54B30; Secondary 18A40. 
Proof. Suppose $X$ is cartesian in Top and take any space $Y$; let $\hat{e}: Y^{X} \times X \rightarrow$ $Y$ be the evaluation map. Consider in $Y^{X}$ the subspace $\mathcal{G}_{\left(X, x_{0}\right)}\left(Y, y_{0}\right)=\{f \in$ $\left.Y^{X} \mid f\left(x_{0}\right)=y_{0}\right\}$ and the restriction $e_{1}$ of $\hat{e}$ to $\mathcal{G}_{\left(X, x_{0}\right)}\left(Y, y_{0}\right) \times\left(X, x_{0}\right)$ which is a

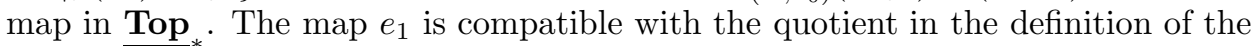
smash-product, and so we can consider the map $e: \mathcal{G}_{\left(X, x_{0}\right)}\left(Y, y_{0}\right) \wedge\left(X, x_{0}\right) \rightarrow\left(Y, y_{0}\right)$ induced by $e_{1}$. We note that $e(f, x)=f(x)$.

Let $f:\left(Z, z_{0}\right) \wedge\left(X, x_{0}\right) \rightarrow\left(Y, y_{0}\right)$ be a map in Top ${ }_{*}$ and consider the quotient $p:\left(Z, z_{0}\right) \times\left(X, x_{0}\right) \rightarrow\left(Z, z_{0}\right) \wedge\left(X, x_{0}\right)$ which gives the smash-product. Since $X$ is cartesian in Top, related to $f p:\left(Z, z_{0}\right) \times\left(X, x_{0}\right) \rightarrow\left(Y, y_{0}\right)$ there is an $(f p)_{1}: Z \rightarrow$ $Y^{X}$ such that $\hat{e}\left((f p)_{1} \times \operatorname{id}_{X}\right)=f p$. The map $(f p)_{1}$ preserves the base points and its image is a subspace of $\mathcal{G}_{\left(X, x_{0}\right)}\left(Y, y_{0}\right)$, so we can factor $(f p)_{1}$ through the inclusion of $\mathcal{G}_{\left(X, x_{0}\right)}\left(Y, y_{0}\right)$ in $Y^{X}$ and consider the first factor $(f p)_{2}$ as a map in Top . $_{*}$. In such a way we obtain $(f p)_{2} \times \operatorname{id}_{X}:\left(Z, z_{0}\right) \times\left(X, x_{0}\right) \rightarrow\left(Y, y_{0}\right)$. By construction $(f p)_{2} \times \mathrm{id}_{X}$ is compatible with the quotient $p$, and the proof is complete.

Theorem 2. If the functor $(-) \wedge\left(X, x_{0}\right)$ has a right adjoint, then $\mathcal{G}_{\left(X, x_{0}\right)}\left(Y, y_{0}\right)$ is a space whose underlying set is in natural bijective correspondence with $\left(Y, y_{0}\right)^{\left(X, x_{0}\right)}$, the counit of the adjunction is the map e: $\mathcal{G}_{\left(X, x_{0}\right)}\left(Y, y_{0}\right) \wedge\left(X, x_{0}\right) \rightarrow\left(Y, y_{0}\right)$ such that $e(f, x)=f(x)$ and the base point corresponds to the constant function valued at $y_{0}$.

Proof. Let $D_{2}$ be the space with two points $a, b$ and the discrete topology. By the adjunction, there is a bijection between $\left(Y, y_{0}\right)^{\left(D_{2}, a\right) \wedge\left(X, x_{0}\right)}$ and $\left(\mathcal{G}_{\left(X, x_{0}\right)}\left(Y, y_{0}\right)\right)^{\left(D_{2}, a\right)}$, and on the other side $\left(D_{2}, a\right) \wedge\left(X, x_{0}\right)$ is homeomorphic to $\left(X, x_{0}\right)$ and $\left(G_{\left(X, x_{0}\right)}\left(Y, y_{0}\right)\right)^{\left(D_{2}, a\right)}$ is in bijection with $\mathcal{G}_{\left(X, x_{0}\right)}\left(Y, y_{0}\right)$; so the first part of the theorem is proved.

Any map $f:\left(X, x_{0}\right) \rightarrow\left(Y, y_{0}\right)$ can be considered as a map from $\left(D_{2}, a\right) \wedge$ $\left(X, x_{0}\right)$ into $\left(Y, y_{0}\right)$. As a consequence, by the adjunction, for any $f$, there is an $f_{1}:\left(D_{2}, a\right) \rightarrow \mathcal{G}_{\left(X, x_{0}\right)}\left(Y, y_{0}\right)$ such that $e\left(f_{1} \wedge \operatorname{id}_{X}\right)=f$; so $e(f, x)=f(x)$. Finally, given the one point space $\bullet$, and the map $h: \bullet \wedge\left(X, x_{0}\right) \rightarrow\left(Y, y_{0}\right)$, there is a map $h_{1}: \bullet \rightarrow \mathcal{G}_{\left.X, x_{0}\right)}\left(Y, y_{0}\right)$ such that $h_{1}(\bullet)$ is the base point of $\mathcal{G}_{\left(X, x_{0}\right)}\left(Y, y_{0}\right)$. This completes the proof.

We denote by $S$ the Sierpinski space with the two points 0 and 1 and $\{0\}$ the nontrivial open set. If the functor $(-) \wedge\left(X, x_{0}\right)$ has a right adjoint, as a consequence of Theorem $2, \mathcal{G}_{\left(X, x_{0}\right)}(S, 0)$ can be identified with the set of the open sets $U$ of $X$ such that $x_{0} \in U$ and base point the open set $X$. On the other hand, $\mathcal{G}_{\left(X, x_{0}\right)}(S, 1)$ can be identified with the set of the open sets $U$ of $X$ such that $x_{0} \notin U$ and base point the empty set.

The following Lemma characterizes convergent nets of the spaces $\mathcal{G}_{\left(X, x_{0}\right)}(S, 0)$ (respectively, $\mathcal{G}_{\left(X, x_{0}\right)}(S, 1)$ ), while Lemma 4 proves that the open sets of these spaces are Scott-open [H-L].

Lemma 3. Suppose $(-) \wedge\left(X, x_{0}\right)$ admits a right adjoint. A net $U_{i}$ converges to $U$ in $\mathcal{G}_{\left(X, x_{0}\right)}(S, 0)$ (respectively, $\mathcal{G}_{\left(X, x_{0}\right)}(S, 1)$ ) if and only if:

$$
\begin{aligned}
& \text { for each } x \in U \text { and for each net } x_{\lambda} \text { converging to } x \text { in } \\
& X \text {, there is an } i^{\prime} \text { and a } \lambda^{\prime} \text { such that } x_{\lambda} \in U_{i} \text {, for every } \\
& i>i^{\prime} \text { and } \lambda>\lambda^{\prime} \text {. }
\end{aligned}
$$

Proof. Let $U_{i}$ converge to $U$ in $\mathcal{G}_{\left(X, x_{0}\right)}(S, 0)$ (respectively, $\left.\mathcal{G}_{\left(X, x_{0}\right)}(S, 1)\right), x \in U$ and $x_{\lambda}$ converge to $x$ in $X$. Consider the counit of the adjunction $e: \mathcal{G}_{\left(X, x_{0}\right)}(S, 0) \wedge$ 
$\left(X, x_{0}\right) \rightarrow(S, 0)$ (respectively, $\left.(S, 1)\right)$ and the quotient map $p: \mathcal{G}_{\left(X, x_{0}\right)}(S, 0) \times$ $\left(X, x_{0}\right) \rightarrow \mathcal{G}_{\left(X, x_{0}\right)}(S, 0) \wedge\left(X, x_{0}\right)$ (respectively, $\left.(S, 1)\right)$. Since $\left(U_{i}, x_{\lambda}\right)$ converges to $(U, x)$ and the map ep is continuous with $e p(U, x)=0$, then $e p\left(U_{i}, x_{\lambda}\right)$ converges to 0 . In $S$ the nets converging to 0 are eventually constant, so there is an $i^{\prime}$ and a $\lambda^{\prime}$ such that, for every $i>i^{\prime}$ and $\lambda>\lambda^{\prime}, e p\left(U_{i}, x_{\lambda}\right)=0$, that is $x_{\lambda} \in U_{i}$.

Vice versa, suppose $U_{i}$ is a net in $\mathcal{G}_{\left(X, x_{0}\right)}(S, 0)$ and $U \in \mathcal{G}_{\left(X, x_{0}\right)}(S, 0)$ (respectively, $\left.\mathcal{G}_{\left(X, x_{0}\right)}(S, 1)\right)$ fulfilling condition $(*)$. Consider the space $I \cup\{\bullet\}$, where $I$ is the direct set of the net $U_{i}, \bullet$ is a maximum point whose base-neighborhoods are the sets of the form $I_{j}=\{\bullet\} \cup\{i \in I \mid i \geq j\}, j \in I$, and the points of $I$ are isolated. We can assume, without changing the nature of the net $U_{i}$, that there exists a point $i_{0} \in I$, such that $U_{i_{0}}=X$ (respectively, $U_{i_{0}}=\emptyset$ ). Moreover, we consider the map $\alpha:\left(I \cup\{\bullet\}, i_{0}\right) \rightarrow \mathcal{G}_{\left(X, x_{0}\right)}(S, 0)$ (respectively, $\left.\mathcal{G}_{\left(X, x_{0}\right)}(S, 1)\right)$ so defined by $\alpha(i)=U_{i}$, $\alpha(\bullet)=U$. We prove that the map $\alpha$ is continuous, which implies the convergence of the net $U_{i}$ to $U$. By the existence of the right adjoint, $\alpha$ is continuous if and only if the corresponding map $e\left(\alpha \wedge \operatorname{Id}_{X}\right)=\bar{\alpha}:\left(I \cup\{\bullet\}, i_{0}\right) \wedge\left(X, x_{0}\right) \rightarrow(S, 0)$ (respectively, $(S, 1))$ is continuous; so we will prove the continuity of $\bar{\alpha}$. To this aim we can consider the quotient map $q:\left(I \cup\{\bullet\}, i_{0}\right) \times\left(X, x_{0}\right) \rightarrow\left(I \cup\{\bullet\}, i_{0}\right) \wedge\left(X, x_{0}\right)$ induced by the smash-product and prove the continuity of $\bar{\alpha} q$. By the adjunction $(\bar{\alpha} q)^{-1}(0)=\left\{(i, x) \mid x \in U_{i}, i \in I\right\} \cup\{(\bullet, x) \mid x \in U\}$. Since every point of $I$ is isolated and each $U_{i}$ is open, the set $\left\{(i, x) \mid x \in U_{i}, i \in I\right\}$ is open since it is union of open sets, therefore each $(i, x)$ of $(\bar{\alpha} q)^{-1}(0)$ belongs to its interior. Take now $(\bullet, x) \in(\bar{\alpha} q)^{-1}(0)$ (that is $\left.x \in U\right)$; the topology defined on $(I \cup\{\bullet\})$ and condition $(*)$ implies that any net converging to $(\bullet, x)$ in the product space $\left(I \cup\{\bullet\}, i_{0}\right) \times\left(X, x_{0}\right)$ is eventually in $(\bar{\alpha} q)^{-1}(0)$, that is, $(\bullet, x)$ belongs to the interior of $(\bar{\alpha} q)^{-1}(0)$. We can conclude that $(\bar{\alpha} q)^{-1}(0)$ is open and so, $\alpha$ is continuous.

Lemma 4. Suppose $(-) \wedge\left(X, x_{0}\right)$ admits a right adjoint, and suppose $H$ is open in $\mathcal{G}_{\left(X, x_{0}\right)}(S, 0)$ (respectively, $\left.\mathcal{G}_{\left(X, x_{0}\right)}(S, 1)\right)$; then $H$ is Scott-open [H-L], i.e.:

(a) If $U, U^{\prime}$ are open in $X, U \in H$ and $U^{\prime} \supseteq U$ (with $x_{0} \notin U^{\prime}$ when $H$ is open in $\left.\mathcal{G}_{\left(X, x_{0}\right)}(S, 1)\right)$, then $U^{\prime} \in H$.

(b) If $V=\left\{U_{i} \mid i \in I\right\}$ is a family of open subsets of $X$ and $U=\bigcup\left\{U_{i} \mid i \in\right.$ $I\} \in H$, there exists a finite subfamily of $V$ whose union belongs to $H$.

Proof. (a) Suppose $U, U^{\prime}$ open in $X, U \in H$ and $U^{\prime} \supseteq U\left(x_{0} \notin U^{\prime}\right.$ in the case when $H$ is open in $\left.\mathcal{G}_{\left(X, x_{0}\right)}(S, 1)\right)$. The constant sequence whose value is $U^{\prime}$ converges to $U$ in $\mathcal{G}_{\left(X, x_{0}\right)}(S, 0)$ (respectively, $\mathcal{G}_{\left(X, x_{0}\right)}(S, 1)$ ) because it fulfils the condition $(*)$ in Lemma 3. Since $H$ is open and $U$ belongs to $H$, there must be an element of the constant sequence belonging to $H$; consequently $U^{\prime} \in H$.

(b) Let $U=\bigcup\left\{U_{i} \mid i \in I\right\} \in H$. If $H$ is open in $\mathcal{G}_{\left(X, x_{0}\right)}(S, 0)$, it follows that $x_{0} \in U$; therefore there exists an $i^{\prime}$ such that $x_{0} \in U_{i^{\prime}}$. Consider in $\mathcal{G}_{\left(X, x_{0}\right)}(S, 0)$ the net whose direct set is $\left\{\left(i_{1}, i_{2}, \ldots, i_{n}\right) \mid i_{1}, i_{2}, \ldots, i_{n} \in I\right\}$ with the relation $\left(i_{1}, i_{2}, \ldots, i_{n}\right) \succ\left(j_{1}, j_{2}, \ldots, j_{m}\right)$ if $U_{i_{1}} \cup U_{i_{2}} \cup \cdots \cup U_{i_{n}} \supseteq U_{j_{1}} \cup U_{j_{2}} \cup \cdots \cup U_{j_{m}}$ and image of $\left(i_{1}, i_{2}, \ldots, i_{n}\right)$ equal to $U_{i^{\prime}} \cup U_{i_{1}} \cup U_{i_{2}} \cup \cdots \cup \bar{U}_{i_{n}}$. This net, according to $(*)$, converges to $U$ in $\mathcal{G}_{\left(X, x_{0}\right)}(S, 0)$; as a consequence, since $H$ is an open set which contains the limit of the net, there exists $\left(i_{1}, i_{2}, \ldots, i_{n}\right)$ such that $U_{i^{\prime}} \cup U_{i_{1}} \cup U_{i_{2}} \cup \cdots \cup$ $U_{i_{n}} \in H$. When $H$ is open in $\mathcal{G}_{\left(X, x_{0}\right)}(S, 1), x_{0} \notin U$ and therefore, $x_{0} \notin U_{i}$, for each $i \in I$. In this case, we can consider the net with the same direct set as above and with the image of $\left(i_{1}, i_{2}, \ldots, i_{n}\right)$ equal to $U_{i_{1}} \cup U_{i_{2}} \cup \cdots \cup U_{i_{n}}$. The same argument of the first case proves that there is $\left(i_{1}, i_{2}, \ldots, i_{n}\right)$ such that $U_{i_{1}} \cup U_{i_{2}} \cup \cdots \cup U_{i_{n}} \in H$. 
Definition 5. A space $X$ is quasi-locally compact if for every $x$ in $X$ and for every neighbourhood $U$ of $x$ there is a neighbourhood of $V \subseteq U$ of $x$ such that every open cover of $U$ has a finite subcover of $V$.

Theorem 6. Let $X$ be a topological space. The following statements are equivalent:

(1) There exists $x_{0}$ in $X$ such that the functor $(-) \wedge\left(X, x_{0}\right)$ : $\mathbf{T o p}_{*} \rightarrow \mathbf{T o p}_{*}$ has a right adjoint.

(2) $X$ is cartesian in Top, that is $X$ is quasi-locally compact.

(3) For any $x_{0}$ in $X$, the functor $(-) \wedge\left(X, x_{0}\right): \mathbf{T o p}_{*} \rightarrow$ Top $_{*}$ has a right adjoint.

Proof. (1) $\rightarrow(2)$ Since the continuity of the counit of the adjunction $e: \mathcal{G}_{\left(X, x_{0}\right)}(S, 0)$ $\wedge\left(X, x_{0}\right) \rightarrow(S, 0)$ implies the continuity of the evaluation map $e^{\prime}: \mathcal{G}_{\left(X, x_{0}\right)}(S, 0) \times$ $\left(X, x_{0}\right) \rightarrow(S, 0)$, it follows that $\left(e^{\prime}\right)^{-1}(0)$ is an open set with $e^{\prime}\left(W, x_{0}\right)=0$, for any $W \in \mathcal{G}_{\left(X, x_{0}\right)}(S, 0)$.

Take a point $x \in X$ and fix $U$ open in $X$ with $x \in U$.

Suppose $x \in \operatorname{cl}\left\{x_{0}\right\}$; then $x_{0} \in U$. Consequently $U$ belongs to $\mathcal{G}_{\left(X, x_{0}\right)}(S, 0)$ and $e^{\prime}(U, x)=0$. Since $\left(e^{\prime}\right)^{-1}(0)$ is open, there is an $H$, open in $\mathcal{G}_{\left(X, x_{0}\right)}(S, 0)$, with $U \in H$ and a neighbourhood $V$ of $x$ such that $\left(e^{\prime}\right)^{-1}(0) \supseteq H \times V$. Since $e^{\prime}(W, y)=0$ when $y \in W$, any element of $H$ contains $V$. Now, consider an open cover of $U$, $\left\{U_{i} \mid i \in I\right\}$ and consider $U^{\prime}=\bigcup\left\{U_{i} \mid i \in I\right\}$. Since $U^{\prime} \supseteq U$, by Lemma 4a), $U^{\prime} \in H$ and by Lemma 4b) there are $i_{1}, i_{2}, \ldots, i_{n} \in I$ such that $U_{i_{1}} \cup U_{i_{2}} \cup \cdots \cup U_{i_{n}} \in H$ and this implies that $U_{i_{1}} \cup U_{i_{2}} \cup \cdots \cup U_{i_{n}} \supseteq V$.

Suppose now $x \notin \operatorname{cl}\left\{x_{0}\right\}$. Therefore, there is an open $A$ of $X$, such that $x \in A$ and $x_{0} \notin A$. The open set $U \cap A \in \mathcal{G}_{\left(X, x_{0}\right)}(S, 1)$; the map $e: \mathcal{G}_{\left(X, x_{0}\right)}(S, 1) \wedge\left(X, x_{0}\right) \rightarrow$ $(S, 1)$ is continuous and $e(U \cap A, x)=0$. Replacing $U^{\prime}=\bigcup\left\{U_{i} \mid i \in I\right\}$ by $U^{\prime}=\bigcup\left\{U_{i} \cap A \mid i \in I\right\}$ in the argument used before proves that there exists a neighbourhood $V$ of $x$ with $U \cap A \supseteq V$, such that any open cover of $U$ (and then of $U \cap A$ ), admits a finite subcover for $V$.

$(2) \rightarrow(3)$ Theorem 1 .

$(3) \rightarrow(1)$ Trivial.

\section{ACKNOWLEDGMENT}

The author wishes to thank the referee, for his helpful suggestion and for this patience.

\section{REFERENCES}

[A] R. Arens, A topology for spaces of transformations, Ann. of Math. (2) 47 (1946), 480-495. MR 8:165e

[A-J] R. Arens and J. Dugundji, Topologies for function spaces, Pacific J. Math. 1 (1951), 5-31. MR 13:264d

[C-F] C. H. Cook and H. R. Fisher, On equicontinuity and continuous convergenge, Math. Ann. 159 (1965), 94-104.

[D] J. Dugundji, Topology, Allyn and Bacon, Boston, MA, 1966. MR 33:1824

[D-K] B. J. Day and G. M. Kelly, On topological quotient maps preserved by pullbacks and products, Math. Proc. Cambridge Philos. Soc. 67 (1970), 553-558. MR 40:8024

[F] R. H. Fox, On topology for function-spaces, Bull. Amer. Math. Soc. 51 (1945), 429-432. MR 6:278d

[H] D. Husemoller, Fiber bundles, McGraw-Hill, New York, 1966. MR 37:4821

$[\mathrm{H}-\mathrm{L}]$ K. H. Hofmann and J. D. Lawson, The spectral theory of distributive continuous lattices, Trans. Amer. Math. Soc. 246 (1978), 285-310. MR 80c:54045

[J] I. M. James, General topology and homotopy theory, Springer-Verlag, Berlin and New York, 1984. MR 86d:55001 
[I] J. R. Isbell, Function spaces and adjoints, Math. Scand. 36 (1975),317-339. MR 53:9134

[Ma] S. Mac Lane, Categories for the working mathematician Graduate Texts in Math., vol. 5, Springer, Berlin and New York, 1971. MR 50:7275

[M] C. R. F. Maunder, Algebraic topology, Cambridge Univ. Press, Cambridge, 1980. MR 84k: 55001

[W] S. Willard, General topology, Addison-Wesley, Reading, MA, 1970. MR 41:9173

Dipartimento di Matematica, Piazza di Porta San Donato, 5, 40127 Bologna, Italy E-mail address: cagliari@dm.unibo.it 